\title{
Influence of heterogeneous and anisotropic tissue conductivity on electric field distribution in deep brain stimulation
}

\author{
Mattias Åström, Jean-Jacques Lemaire and Karin Wårdell
}

\section{Linköping University Post Print}

N.B.: When citing this work, cite the original article.

The original publication is available at www.springerlink.com:

Mattias Åström, Jean-Jacques Lemaire and Karin Wårdell, Influence of heterogeneous and anisotropic tissue conductivity on electric field distribution in deep brain stimulation, 2012, Medical and Biological Engineering and Computing, (50), 1, 23-32.

http://dx.doi.org/10.1007/s11517-011-0842-z

Copyright: Springer Verlag (Germany) http://www.springerlink.com/

Postprint available at: Linköping University Electronic Press http://urn.kb.se/resolve?urn=urn:nbn:se:liu:diva-70087 


\title{
Influence of heterogeneous and anisotropic tissue
}

\section{conductivity on electric field distribution in deep brain}

\section{stimulation}

\author{
Mattias Åström $^{1}$, Jean-Jacques Lemaire ${ }^{2,3}$, and Karin Wårdell ${ }^{1}$ \\ ${ }^{1}$ Department of Biomedical Engineering, Linköping University, Sweden \\ ${ }^{2}$ Clermont Université, Université d'Auvergne, EA 3295, Equipe de recherche en signal et \\ imagerie médicale, Image-Guided Clinical Neuroscience and Connectomics (IGCNC), BP \\ 10448, F-63000 Clermont-Ferrand, France \\ ${ }^{3}$ CHU Clermont-Ferrand, Service de Neurochirurgie, F-63003 Clermont-Ferrand, France
}

\section{Corresponding author}

Mattias Åström

Department of Biomedical Engineering

Linköping University

58185 Linköping, Sweden

E-mail: matas@imt.liu.se

Phone: +46 (0) 736290978

Total number of words: 6725

Number of words in abstract: 194

Number of figures: 5

Number of tables: 4

\section{Keywords}

Deep brain stimulation; diffusion tensor; finite element; model; simulation; patient-specific; 


\section{Abstract}

The aim was to quantify the influence of heterogeneous isotropic and heterogeneous anisotropic tissue on the spatial distribution of the electric field during deep brain stimulation (DBS). Three finite element tissue models were created of one patient treated with DBS. Tissue conductivity was modelled as I) homogeneous isotropic, II) heterogeneous isotropic based on MRI, and III) heterogeneous anisotropic based on diffusion tensor MRI. Modelled DBS electrodes were positioned in the subthalamic area, the pallidum, and the internal capsule in each tissue model. Electric fields generated during DBS were simulated for each model and target-combination and visualized with isolevels at 0.20 (inner), and $0.05 \mathrm{~V} \mathrm{~mm}^{-1}$ (outer). Statistical and vector analysis was used for evaluation of the distribution of the electric field. Heterogeneous isotropic tissue altered the spatial distribution of the electric field by up to $4 \%$ at inner, and up to $10 \%$ at outer isolevel. Heterogeneous anisotropic tissue influenced the distribution of the electric field by up to $18 \%$ and $15 \%$ at each isolevel, respectively. The influence of heterogeneous and anisotropic tissue on the electric field may be clinically relevant in anatomic regions that are functionally subdivided and surrounded by multiple fibres of passage. 


\section{Introduction}

Deep brain stimulation (DBS) is a widely used therapy for selected patients with severe Parkinson's disease (PD), essential tremor, and primary dystonia. The overall clinical outcome of DBS depends on several biological variables such as severity of disease, duration of disease since the onset of symptoms, type of symptoms, and drug sensibility [1-3]. DBS acts as a local delivery treatment and so is dependent on the volume of tissue influenced by the stimulation [4]. Thus, knowledge of the anatomical distribution of electric field generated during DBS is crucial for maximizing the therapeutic effects of this therapy. Several technical aids for pre, intra and post surgery are currently being developed and enhanced [5]. An example is patient-specific computer simulations of the electric field

generated during DBS [6, 7]. Åström et al., have previously used pre- and postoperative MRI acquired during the planning and the postoperative follow-up of the surgical DBS procedure for creating patient-specific models and simulations of DBS $[6,8]$. Brain tissue is classified in the MRI and material properties from the literature are allotted to each classified MRI-voxel. However, models based on regular MRI neglect the directional dependency, the anisotropy, of the electrical conductivity of brain tissue. The impedance of myelinated fibre bundles in the internal capsule of a cat has been measured to be approximately nine times greater perpendicular to the axonal fibres than along these fibres [9]. Sotiropoulos and Steinmetz [10] incorporated non patient-specific heterogeneous and anisotropic tissue for simulations of DBS in the subthalamic nucleus (STN). It has been suggested by Tuch and others [11-13] that diffusion tensor magnetic 
resonance imaging (DTI) can be used to calculate anisotropic electrical conductivity tensors in patient-specific anatomy. The basis for a general relationship between diffusion of water and electrical conductivity in biological tissue is the mutual geometrical restrictions for both ionic and water mobility [12]. Because of the effective electrical shielding of the neuronal membranes, both the electric conductivity and water diffusion are primarily mediated in the extracellular pathways. This predicts a linear scaling relationship between water diffusion and electrical conductivity. Walackier et al. [14] have previously created DBS models based on patient DTI, and Butson et al. created a finite element tissue model based on a DTI atlas that has been fitted to patient-specific anatomy $[7,15]$. This approach has recently been used to study the influence of different model complexity on neural activation [16]. In the present study patient-specific stereotactic MRI, and DTI acquired during the surgical DBS procedure was used to create patient-specific models of the brain. The overall aim was to quantify the influence of heterogeneous isotropic and heterogeneous anisotropic tissue on the spatial distribution of the electric field during DBS by means of patient specific models and simulations.

\section{Materials and methods}

\section{Tissue models}

Three finite element tissue models were created in three deep brain anatomic regions of one patient suffering from severe Parkinson's disease. The patient was 


\section{Influence of tissue on DBS}

undergoing bilateral DBS surgery in the subthalamic region. Stereotactic preoperative MRI, including DTI, and postoperative computed tomography (CT) scans were used during the modelling process. Preoperative T2-weighted MRI and DTI were acquired one day before surgery on a 1.5 Tesla machine (Sonata, Siemens, Germany) with a voxel volume of $0.56 \mathrm{~mm}^{3}(0.53 \times 0.53 \times 2)$ and 9.72 $\mathrm{mm}^{3}(1.8 \times 1.8 \times 3)$, respectively. The acquisition parameters for the T2-weighted MRI were: repetition time, $4500 \mathrm{~ms}$; echo time, $13 \mathrm{~ms}$; and number of averages, 2. Acquisition parameters for the DTI were: repetition time, $3300 \mathrm{~ms}$; echo time, $84 \mathrm{~ms}$; number of averages, 7 ; number of b0 images 1; and number of gradient directions, 6. The postoperative CT were acquired on a LightSpeed QX/i (GE Medical Systems, USA) with a voxel volume of $0.08 \mathrm{~mm}^{3}(0.43 \times 0.43 \times 0.43)$.

In Model I the tissue-medium was modelled as a homogeneous isotropic volume conductor with an electric conductivity of $0.075 \mathrm{~S} \mathrm{~m}^{-1}$ (Siemens metre ${ }^{-1}$ ) representing a mixture of white and grey matter [17]. This model was regarded as a baseline model for comparison with the two other models. In Model II the methods previously developed by our group was followed $[6,8]$. The tissue was modelled as heterogeneous and isotropic based on preoperative T2 weighted stereotactic MRI. The preoperative MRI voxels were classified according to the intensity of each voxel and were allotted electrical conductivity properties from Andreuccetti's online database [17]. The electrical conductivity of cerebrospinal fluid was set to $2.00 \mathrm{~S} \mathrm{~m}^{-1}$, grey matter $0.09 \mathrm{~S} \mathrm{~m}^{-1}$, white matter $0.06 \mathrm{~S} \mathrm{~m}^{-1}$, and blood $0.70 \mathrm{~S} \mathrm{~m}^{-1}$. Because of partial volume effects a linear interpolation function was used to allot approximated electrical physical properties to voxels containing more than one tissue. The result was a matrix of electrical tissue properties located 
at the centre of each voxel. Tissue conductivities were interpolated between the centres of each voxel for a continuous representation of the electrical properties of the tissue-medium. In Model III the tissue-medium were modelled as heterogeneous and anisotropic based on preoperative DTI. Each second order diffusion tensor was represented by a symmetric positive definite 3 by 3 matrix, D:

$$
\boldsymbol{D}=\left[\begin{array}{lll}
D_{x x} & D_{x y} & D_{x z} \\
D_{x y} & D_{y y} & D_{y z} \\
D_{x z} & D_{y z} & D_{z z}
\end{array}\right]
$$

where the subscripts describes each direction. The tensors were extracted from the DTI dataset using the software DtiStudio Version 2.40 [18]. Tuch et al. [11, 12] showed a strong cross-property relation between the electrical conductivity and water self-diffusion tensors in extracellular brain tissue:

$$
\boldsymbol{\sigma}=\frac{\sigma_{e}}{d_{e}} \boldsymbol{D} \quad\left[S m^{-1}\right]
$$

where $\sigma$ is the electrical conductivity tensor, $\sigma_{\mathrm{e}}\left(\mathrm{S} \mathrm{m}^{-1}\right)$ is the effective extracellular electrical conductivity, $d_{\mathrm{e}}\left(\mathrm{m}^{2} \mathrm{~s}^{-1}\right)$ is the effective extracellular diffusivity, and $\mathrm{D}$ is the diffusion tensor. The intracellular conductivity was taken as negligible because of the effective shielding by the high resistivity of the cell membrane for frequencies in the DBS range $(130-185 \mathrm{~Hz}<1 \mathrm{kHz})$. In the present study the ratio of $\sigma_{\mathrm{e}} / d_{\mathrm{e}}$ was set to $0.844 \mathrm{Ss} / \mathrm{mm}^{3}$ as empirically derived by Tuch et al. 2001 [11]. The electrical conductivity tensors were mapped in the finite element model at coordinates corresponding to their original location in the DTI data set. The result was a property matrix with the same resolution as the DTI data 
set containing electrical anisotropic conductivity tensors. The three tissue models' characteristics are summarised in Table 1.

Table 1. Characteristics of the three finite element tissue models. The tissuemedium were modelled as (I) homogeneous isotropic, (II) heterogeneous and isotropic based on voxel classification of preoperative T2-weighted MRI, and (III) heterogeneous and anisotropic based on linearly scaled preoperative DTI.

\begin{tabular}{|c|c|c|}
\hline Model & Electrical conductivity $\left[\mathrm{S} \mathrm{m}^{-1}\right]$ & $\begin{array}{l}\text { Tissue-medium } \\
\text { characteristics }\end{array}$ \\
\hline I & 0.075 & $\begin{array}{l}\text { Homogenous and } \\
\text { isotropic }\end{array}$ \\
\hline II & $\mathrm{MRI}$ and literature & $\begin{array}{l}\text { Heterogeneous and } \\
\text { isotropic }\end{array}$ \\
\hline III & $\mathrm{DTI} \times 0.844$ & $\begin{array}{l}\text { Heterogeneous and } \\
\text { anisotropic }\end{array}$ \\
\hline
\end{tabular}

\section{Anatomic regions}

Two DBS electrodes with a radius of $0.635 \mathrm{~mm}$ and contact lengths of $1.5 \mathrm{~mm}$ separated by $0.5 \mathrm{~mm}$, simulating a 3389 DBS $^{\mathrm{TM}}$ Lead (Medtronic, Inc. USA), were modelled and positioned bilaterally in three anatomic regions of the brain: the dorso-lateral border of the subthalamic nucleus (denoted SubT), the posterodorsal part of the internal pallidum (GPi), and the foremost part of anterior limb of the internal capsule (IC). These three regions correspond to three functional targets for treatement with DBS in Parkinson's disease, dystonia, and obsessivecompulsive disorders, respectively.

Within the subthalamus, the DBS electrodes were visible as artefacts on the postoperative CT. These artefacts were used to position the modelled electrodes at their patient-specific locations at the dorso-lateral border of the STN. The 


\section{Influence of tissue on DBS}

modelled electrodes were positioned in the centre of the electrode artefacts after co-registration of the postoperative CT with the preoperative MRI and DTI using Iplan (BrainLab, Germany). A detailed explanation on patient-specific electrode placement can be found in Astrom et al. 2009 [6].

Within the postero-dorsal part of the GPi and the foremost part of anterior limb of the IC, modelled electrodes were manually placed. Thus, for these two targets the models were not patient-specific regarding the location of the electrodes. The positions of electrodes remained fixed between the different tissue model types. As a result, the locations and orientations of the electrodes were identical in relation to patient-specific anatomy between the three tissue models.

\section{Simulations}

The electric field was simulated for model I, II, and III, in each of the three anatomic regions (SubT, GPi, and IC) resulting in nine simulations in total. Monopolar electrode configuration was simulated bilaterally in each target where contacts 1 and 5 (contacts located just above the distal contacts on the right and left side) were used as cathodes with an electric potential of $-3 \mathrm{~V}$. Simulations were carried out with Comsol Multiphysics version 3.5 (Comsol Multiphysics $\mathrm{AB}$, Sweden). The distribution of the electric field in the vicinity of the active contacts was calculated using the equation of continuity for steady currents [19]:

$$
\nabla \cdot \mathrm{J}=-\nabla \cdot[\sigma \nabla \mathrm{V}]=0 \quad\left(A m^{-3}\right)
$$

where $J$ is the current density $\left(\mathrm{A} \mathrm{m}^{-2}\right), \sigma$ the electrical conductivity $\left(\mathrm{S} \mathrm{m}^{-1}\right)$, and $V$ the electric potential (V). The models were solved on a 64-bit Linux computer 


\section{Influence of tissue on DBS}

(3.6 GHz Intel Xeon processor, 16 GB RAM) for 2,500,000 degrees of freedom using Comsol Multiphysics' iterative linear system solver GMRES with the preconditioner Incomplete LU.

\section{Visualization}

The electric field was visualized in 3D with isolevels at $0.20 \mathrm{~V} \mathrm{~mm}^{-1}$ and $0.05 \mathrm{~V}$ $\mathrm{mm}^{-1}$ denoted inner and outer isolevel, respectively. These isolevels were chosen in order to display the electric field at two different radial distances away from the active electrode contact of approximately 2.7 and $6.1 \mathrm{~mm}$ during stimulation at 3 V. The electric field was visualized together with anatomic information on twodimensional axial and coronal slices. The intersections of the electric field isolevels with the MRI slices were traced. Each trace was colour-coded according to tissue model type where Model I was white, Model II was red, and Model III was yellow (Figure 1).

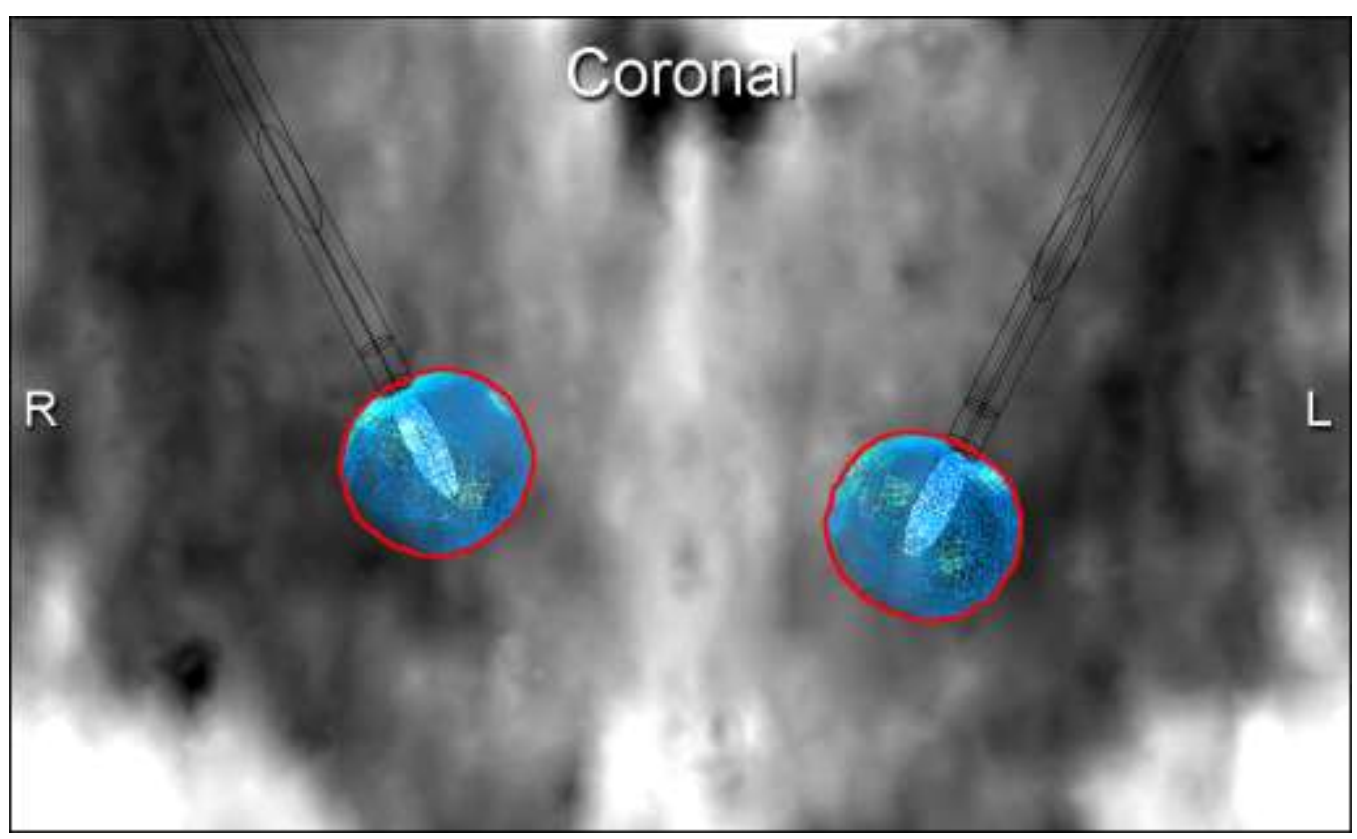

Figure 1. Electric field and MRI anatomy. Frontal view of the anatomy together with the electric field isolevel at $0.20 \mathrm{~V} \mathrm{~mm}^{-1}$ (green) on reconstructed coronal MRI slice. The intersection of the electric field isolevel and the coronal slice was traced and colour-coded according to tissue model type. Here, the electric field isolevel of tissue Model II was simulated and traced in red. 


\section{Influence of tissue on DBS}

The tissue-micro structure was visualized with diffusion tensor data together with the MRI. A software tool was developed in Matlab 7.0 (The MathWorks, USA) for visualization of diffusion tensors with superquadric glyphs as described by Kindlmann (2004) [20] and later by Ennis et al. (2005) [21]. The diffusion tensors were decomposed from the 3 by 3 matrix, D (Eq. 1), into a local eigensystem of three mutually orthogonal eigenvectors and three eigenvalues. The eigenvalues were parameterized into linear, planar, and spherical anisotropy, $\mathrm{C}_{\mathrm{L}}, \mathrm{C}_{\mathrm{P}}$, and $\mathrm{C}_{\mathrm{S}}$, respectively [22]:

$$
C_{L}=\frac{\lambda_{1}-\lambda_{2}}{\lambda_{1}+\lambda_{2}+\lambda_{3}}, C_{P}=\frac{2\left(\lambda_{2}-\lambda_{3}\right)}{\lambda_{1}+\lambda_{2}+\lambda_{3}}, C_{S}=\frac{3 \lambda_{3}}{\lambda_{1}+\lambda_{2}+\lambda_{3}}
$$

where the eigenvalues, $\lambda$, were sorted in descending order so that $\lambda_{1} \geq \lambda_{2} \geq \lambda_{3} \geq 0$. The shapes of the glyphs were then derived from two different parametric equations depending of the anisotropy of the tensor Eq. 7 in [20]:

$$
\begin{aligned}
& \mathrm{C}_{\mathrm{L}} \geq \mathrm{C}_{\mathrm{p}} \Rightarrow\left\{\begin{array}{l}
\alpha=\left(1-C_{P}\right)^{\gamma}, \beta=\left(1-C_{L}\right)^{\gamma} \\
p(\theta, \emptyset)=\left(\begin{array}{c}
\cos ^{\beta} \emptyset \\
-\sin ^{\alpha} \theta \sin ^{\beta} \emptyset \\
\cos ^{\alpha} \theta \sin ^{\beta} \emptyset
\end{array}\right)
\end{array}\right. \\
& \mathrm{C}_{\mathrm{L}}<\mathrm{C}_{\mathrm{p}} \Rightarrow\left\{\begin{array}{c}
\alpha=\left(1-C_{L}\right)^{\gamma}, \beta=\left(1-C_{P}\right)^{\gamma} \\
p(\theta, \emptyset)=\left(\begin{array}{c}
\cos ^{\alpha} \theta \sin ^{\beta} \emptyset \\
\sin ^{\alpha} \theta \sin ^{\beta} \emptyset \\
\cos ^{\beta} \emptyset
\end{array}\right)
\end{array}\right. \\
& 0 \leq \emptyset \leq \pi \\
& 0 \leq \theta \leq 2 \pi
\end{aligned}
$$

where all the exponentials are signed: $x^{a}=\operatorname{sign}(x)|x|^{a}$. The parameter $\gamma$ 


\section{Influence of tissue on DBS}

highlights the anisotropy of the tensor by affecting the sharpness of the edges of the glyphs. In the present study $\gamma$ was empirically chosen to 3 for creating glyphs with rounded edges that allowed a clear and intuitive view of the orientation of the glyph. The orientation of the glyphs was determined by the eigenvectors. Each glyph was colour-coded according to its deviation from the isotropic case, $\mathrm{C}_{\mathrm{A}}$ [22]:

$$
C_{A}=1-C_{S}
$$

Where a high value of $\mathrm{C}_{\mathrm{A}}$ was coloured red and a low value was coloured blue (Figure 2).

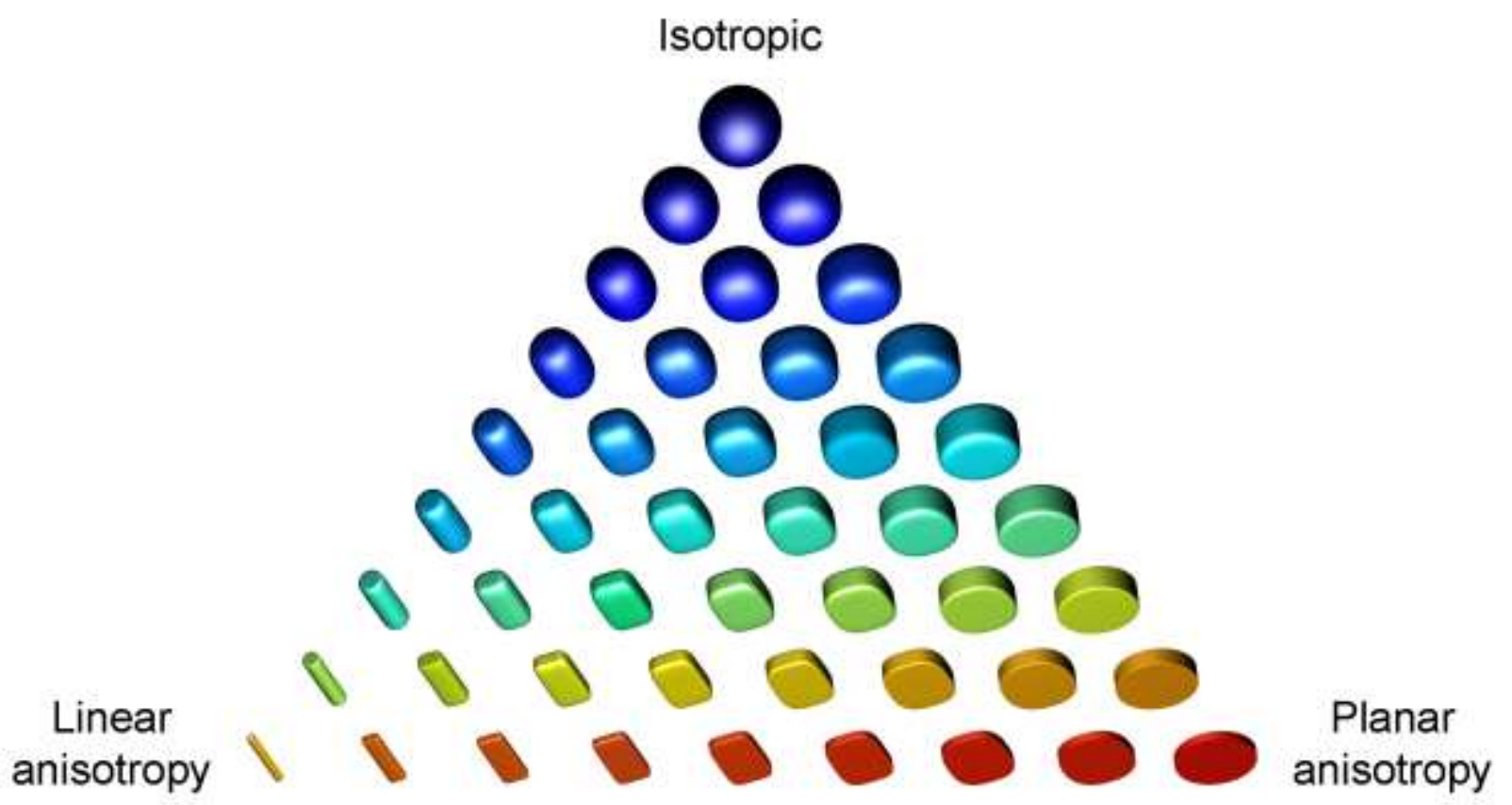

Figure 2. Superquadric glyphs. The shape of the glyphs represented the tissue anisotropy, where a cylindrical shape reflected linear anisotropy, a disk shape reflected planar anisotropy, and a spherical shape reflected isotropic tissue. The glyphs were colour-coded according to their deviation from the isotropic case where blue was isotropic and red was highly anisotropic.

\section{Data analysis}

The spatial distribution of the electric field was quantified and compared between baseline model (Model I), and Model II and III. The diameters of the simulated 
electric field isolevels at $0.20 \mathrm{~V} \mathrm{~mm}^{-1}$ and $0.05 \mathrm{~V} \mathrm{~mm}^{-1}$ were measured in 9 orientations: $\mathrm{x}, \mathrm{xy}, \mathrm{xz},-\mathrm{xy}, \mathrm{y}, \mathrm{yz},-\mathrm{xz},-\mathrm{yz}$, and $\mathrm{z}$, where $\mathrm{x}$ was medial-lateral; $\mathrm{y}$ was anterior-posterior; and z was superior-inferior (Figure 3). Because of bilateral placement of DBS electrodes, 18 measurements were carried out for each isolevel, anatomic region, and model combination. Since the modelled electrodes were positioned with the same angle and location in each anatomic region, each diameter could be directly compared between models. Evaluations of the distribution of the electric field isolevels were performed using vector analysis and descriptive statistics.

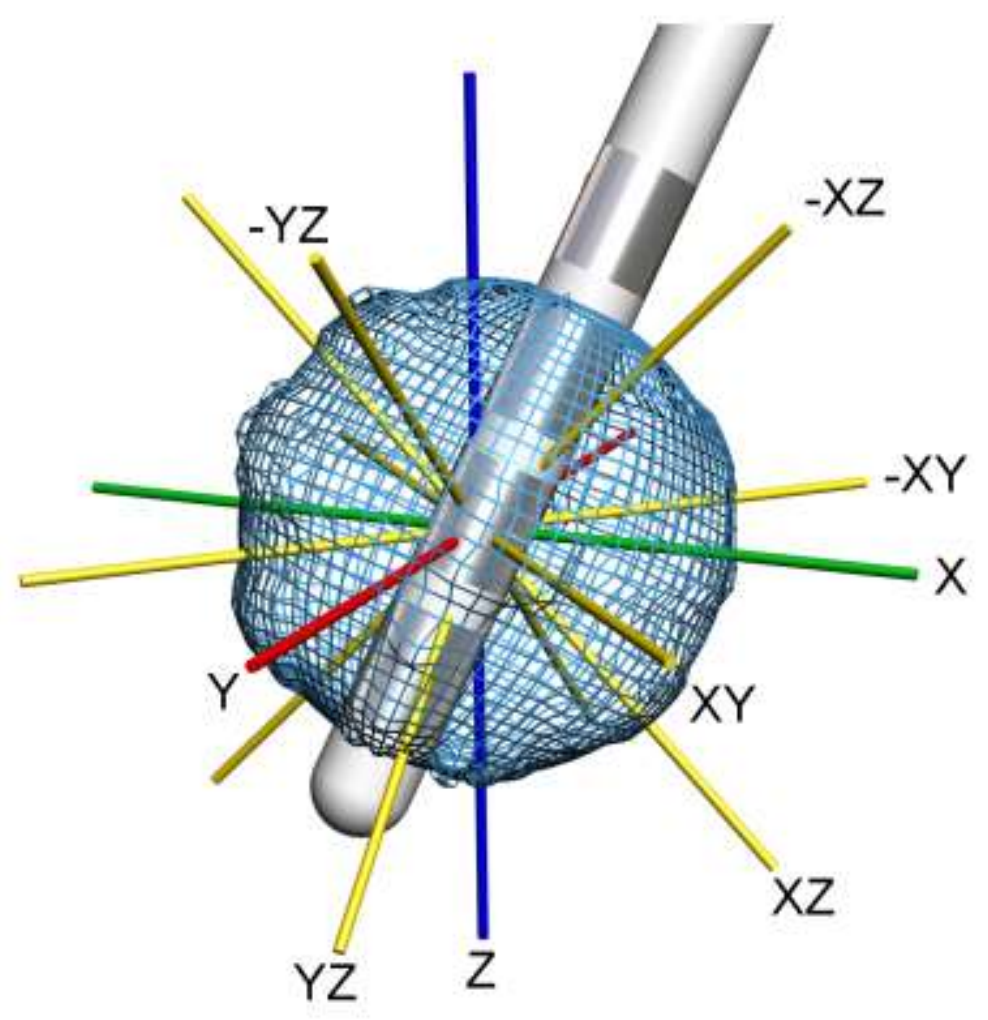

Figure 3. Modeled DBS-electrode with animated electric field isolevel in wireframe. The distance to the electric field isolevels were measured in nine directions; $\mathrm{x}, \mathrm{y}, \mathrm{z}, \pm \mathrm{xy}, \pm \mathrm{xz}$, and $\pm \mathrm{yz}$, where $\mathrm{x}$ was oriented medial-lateral; $\mathrm{y}$ anterior-posterior; and $\mathrm{z}$ inferior-superior in relation to the plane of the anterior and posterior commissure. An electric field isolevel was animated with wireframe. 
Vector analysis was performed to derive a scalar value of the overall alteration of the shape of the electric field isolevels between baseline model, and Model II and III. The 18 bilateral measured diameters from each isolevel, anatomic region, and model combination were used to create 18 -dimensional vectors. An angle, $v$, between each baseline model vector, $A$, and the corresponding vector of Model II and III, $B$, were calculated based on the law of cosines.

$$
v=\arccos \left(\frac{A^{T} B}{\sqrt{A^{T} A \cdot B^{T} B}}\right)
$$

The angle, $v$, which was displayed in degrees, represented a measure of the

electric field shape differences between the models, where a large angle represented a large difference. The vector analysis was performed using MatLab 7.0 (MathWorks Inc., USA).

Descriptive statistics was used to calculate the mean and standard deviation (StDev) of the measured diameters for each isolevel, anatomic region, and model combination. In addition, the diameters of Model II and III were directly compared with the corresponding diameters of baseline-model by calculating the difference between each corresponding diameter. Mean, standard deviation, minimum, and maximum of the differences were calculated for Model II and III. The minimum and maximum values were also expressed as a percentage in relation to the mean diameter length of the baseline model diameters. Statistical calculations were performed with Minitab (Minitab Inc., USA) and Microsoft Excel (Microsoft Inc., USA). 


\section{Results}

Patient-specific MRI and DTI data were visualized on axial and coronal slices together with contours of the inner and outer isolevels in the SubT, GPi, and IC (Figure 4). We found that the spatial distribution of the electric field was altered by the heterogeneous isotropic (Model II) and heterogeneous anisotropic (Model III) tissue compared to baseline model (Model I). In general, vector analysis showed that the shape of the electric field isolevel was altered to a smaller extent in Model II than in Model III (Table 2). In tissue Model II the mean angles for all anatomic regions were 0.8 , and 1.8 degrees at the inner and outer isolevels, respectively. In Model III the corresponding angles were 3.1 and 3.0 degrees. The influence of heterogeneous and anisotropic tissue on the electric field varied in different anatomic regions. Vector analysis showed largest angles in GPi for both Model II and III, at both isolevels.

Table 2. The diameters of each anatomic region and model combination were regarded as 18-dimensional vectors. Angles in degrees were calculated between corresponding vectors of Model II and III, and baseline model (Model I). Large angles correspond to large differences in the shape of the electric field compared to baseline model.

\begin{tabular}{ccccc}
\hline & \multicolumn{4}{c}{ Angle $\left[^{\circ}\right]$} \\
Model & Anatomic region & Iso 0.20 & Iso 0.05 & $\mathrm{n}$ \\
\hline II & STN & 0.8 & 1.9 & 18 \\
II & GPi & 1.2 & 2.3 & 18 \\
II & IC & 0.6 & 1.3 & 18 \\
III & STN & 2.6 & 3.4 & 18 \\
III & GPi & 4.2 & 3.6 & 18 \\
III & IC & 2.5 & 1.9 & 18 \\
\hline
\end{tabular}



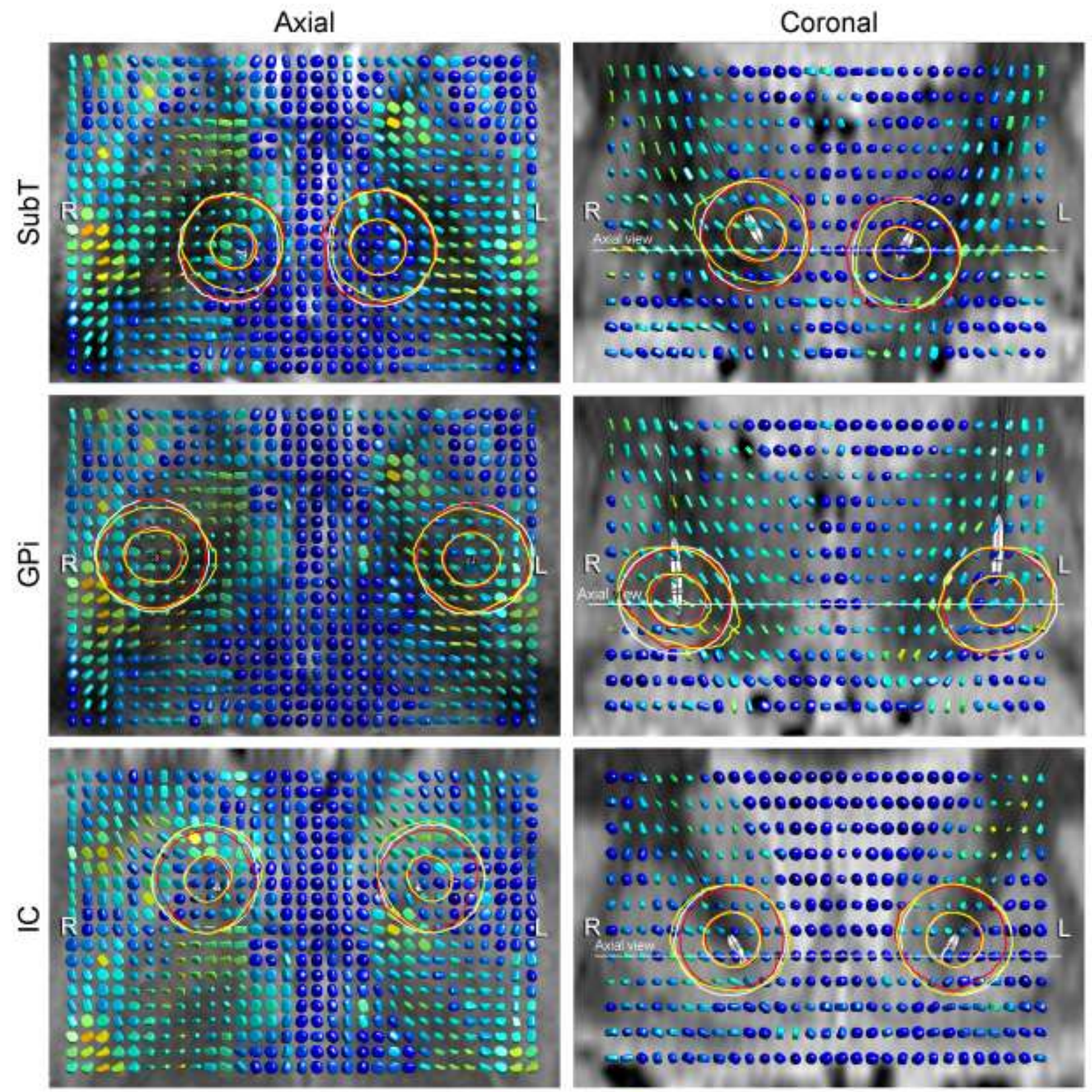

aModel I Model II Model III

Figure 4. Simulated electric field isolevels at $0.20 \mathrm{~V} \mathrm{~mm}^{-1}$ and $0.05 \mathrm{~V} \mathrm{~mm}^{-1}$ presented on axial (left column) and coronal (right column) MRI slices. Simulations were performed within the dorolateral-STN-subthalamic-area (SubT), pallidum (GPi) and the foremost part of anterior limb of the internal capsule (IC) for tissue Model I (white), Model II (red) and Model III (yellow). DTI was displayed with colour-coded superquadric glyphs from blue, isotropic, to red highly anisotropic. The superquadric glyphs were intersected at their centre by the MRI slice showing the structural anatomy. The level of the axial and coronal MRI and superquadric glyphs was chosen as a compromise between closest to the active electrode contacts of the left and right electrodes. 


\section{Influence of tissue on DBS}

In agreement with the vector analysis, the descriptive statistics showed lower standard deviations of the measured diameters in Model II than in Model III (Table 3). In tissue Model II the mean standard deviation for all anatomic regions were 0.12 , and $0.41 \mathrm{~mm}$ at the inner and outer isolevels, respectively. In Model III the corresponding values were 0.39 and $0.75 \mathrm{~mm}$.

Table 3. Mean and standard deviation of the length $(\mathrm{mm})$ of the measured electric field isolevel diameters for each model and target combination.

\begin{tabular}{ccccc}
\hline & \multicolumn{4}{c}{ Mean diameter (StDev), mm } \\
Model & Anatomic region & Iso 0.20 & Iso 0.05 & n \\
\hline I & STN & $6.63(0.12)$ & $13.32(0.35)$ & 18 \\
I & GPi & $6.71(0.18)$ & $13.36(0.31)$ & 18 \\
I & IC & $6.69(0.11)$ & $13.31(0.20)$ & 18 \\
II & STN & $6.73(0.11)$ & $13.57(0.41)$ & 18 \\
II & GPi & $6.75(0.16)$ & $12.93(0.51)$ & 18 \\
II & IC & $6.73(0.09)$ & $12.71(0.32)$ & 18 \\
III & STN & $6.74(0.33)$ & $12.78(0.68)$ & 18 \\
III & GPi & $6.82(0.52)$ & $13.11(0.98)$ & 18 \\
III & IC & $6.94(0.33)$ & $13.57(0.59)$ & 18 \\
\hline
\end{tabular}

The diameters of Model II and III were directly compared with the corresponding diameters of baseline model by calculating the mean, minimum, and maximum of the diameters differences (Table 4). In general, Model II showed smaller diameters differences than Model III. The minimum and maximum differences of Model II were $(-0.2,0.3)$ and $(-1.3,1.1) \mathrm{mm}$ at the inner and outer isolevels, respectively. Expressed as a percentage of the mean diameter of baseline model for each isolevel (6.7 $\mathrm{mm}$ and $13.4 \mathrm{~mm}$, respectively) this corresponded to (-3\%, $4 \%)$ and $(-10 \%, 8 \%)$. In Model III the corresponding values were $(-0.5,1.2)$, and $(-1.8,2.0) \mathrm{mm}$. Expressed as a percentage this corresponded to $(-7 \%, 18 \%)$, and ($13 \%, 15 \%)$. 
Table 4. Mean, minimum, and maximum diameter differences $(\mathrm{mm})$ between baseline model and Model II, and III.

\begin{tabular}{ccccc}
\hline & & \multicolumn{3}{c}{ Mean difference (Min, Max), mm } \\
Models & Anatomic region & Iso 0.20 & Iso 0.05 & $\mathrm{n}$ \\
\hline II-I & STN & $0.11(-0.1,0.3)$ & $0.25(-0.4,1.1)$ & 18 \\
II-I & GPi & $0.04(-0.2,0.3)$ & $-0.43(-1.3,0.9)$ & 18 \\
II-I & IC & $0.03(-0.1,0.2)$ & $-0.60(-1.0,0.1)$ & 18 \\
III-I & STN & $0.12(-0.3,0.7)$ & $-0.54(-1.8,1.0)$ & 18 \\
III-I & GPi & $0.11(-0.5,1.2)$ & $-0.25(-1.3,2.0)$ & 18 \\
III-I & IC & $0.24(-0.2,0.8)$ & $0.26(-0.4,1.0)$ & 18 \\
\hline
\end{tabular}

In Model III, anisotropic tissue had a marked directional influence on the electric field. Largest diameter difference compared to baseline model was 1.2 and 2.0 $\mathrm{mm}$ at the inner and outer isolevels, and was found in the right GPi parallel to the internal capsule, direction xz. The alterations of the electric field were related to the tissue micro-structure as presented by the superquadric glyphs. Because of linear anisotropy in this region, the electric field was further extended in the direction of the main axis of the conductivity tensor, and compressed perpendicular to the main axis (Figure 5).

\section{Discussion}

In the present study the influence of heterogeneous and anisotropic tissue on the spatial distribution of the electric field during DBS was quantified and compared by means of patient-specific computer models and simulations in realistic DBS targets. The inner and outer isolevel $\left(0.20\right.$ and $\left.0.05 \mathrm{~V} \mathrm{~mm}^{-1}\right)$ were altered (reduced or extended) in various directions compared to baseline model. In Model II, the alteration of the inner and outer isolevel diameter in various directions was up to $4 \%$ and $10 \%$, respectively. In Model III the corresponding values were up to $18 \%$ and $15 \%$, respectively. 


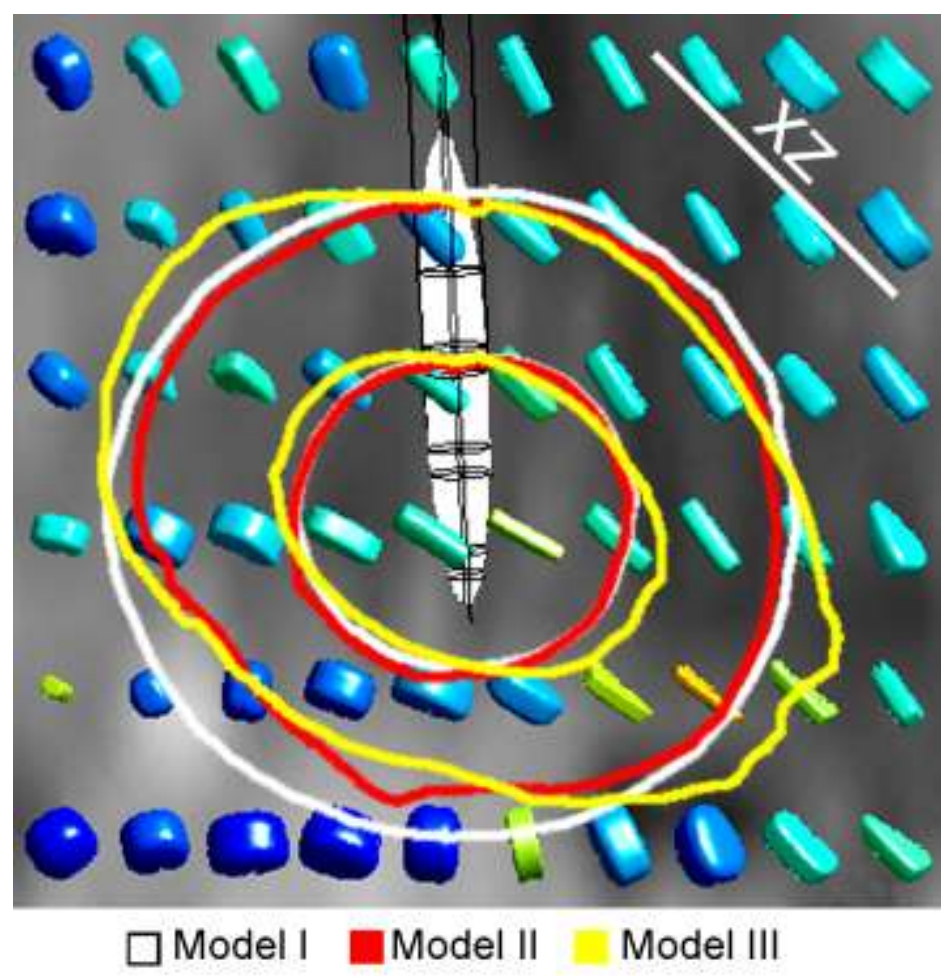

Figure 5. Coronal view of the electric field isolevels at 0.20 and $0.05 \mathrm{~V} \mathrm{~mm}^{-1}$ together with superquadric glyphs and MRI in the right GPi. In model III, which was based on DTI, the electric field was further extended in the direction of the main axis of the superquadric glyphs and compressed perpendicular to the main axis.

Electric field isolevels were visualized and not the response of neural tissue as calculated by the activating function [23] commonly used by others [7, 16, 24].

This was an intentional choice in order to present the results in a general manner without restricting the interpretation of the results to specific axon orientations and diameters, along with additional assumptions regarding the electrode encapsulation tissue [25] and the voltage drop at the electrode-tissue interface [26].

The effect on the electric field was specific to each anatomic region and also to each hemisphere. A combination of MRI and DTI data together with simulated electric field provided a detailed visual feed-back of anatomy, microstructure and 


\section{Influence of tissue on DBS}

electric field during DBS. In Model III, the distribution of the electric field was finely tuned with respect to the tissue microstructure as presented by the superquadric glyphs (Figure 5). Thus, the orientation and shape of the superquadric glyphs may provide an indication of the spatial distribution of the electric field, even without simulations of the electric field.

The electric field was measured at two different isolevels, inner and outer, in order to evaluate the influence of heterogeneous and anisotropic tissue on the electric field at different distances away from the electrode. The inner and outer isolevels were located approximately $2.7 \mathrm{~mm}$ and $6.1 \mathrm{~mm}$ away from the surface of the active electrode contact. Although the exact volume of neural tissue influenced during DBS is not known, the radial distance to the outer isolevel is considered to be outside the region where the major effect is taking place during stimulation at $3 \mathrm{~V}[27,28]$. The distance to the inner isolevel, however, may be regarded as within or at the border of the region of influence during standard DBS settings [29]. Thus, although the influence of heterogeneous and anisotropic tissue on the electric field was generally in the sub-millimetre level there were cases where the electric field diameter was altered by up to $1.2 \mathrm{~mm}$ at the inner isolevel (Table 4). Such alterations may possibly have an impact on the clinical outcome during DBS because of modulation of the activity of neurons within the targeted nucleus as well as neighbouring myelinated fibres. This could be the case when targeting the subthalamus area, where STN is subdivided into different functional parts and surrounded and crossed by several fibre paths such as the ansa lenticularis and the superior thalamic radiations. In comparison it is well known that changing the active contact of Medtronic's model 3389 DBS $^{\text {TM }}$ Lead to a more superior or 


\section{Influence of tissue on DBS}

inferior contact, and thereby moving the current source by $2 \mathrm{~mm}$, may result in a different clinical outcome.

The analysis of the effect of heterogeneous isotropic and heterogeneous anisotropic tissue on the spatial distribution of the electric field during DBS was limited by several reasons. Only nine diameters were measured of each electric field isolevel which does not provide a full representation of its spatial distribution. Patient-specific models based on DTI [30, 31] or MRI [8] have already been used in clinical investigations. However, the realism of models based on DTI or MRI are limited by several, but different factors. The major limitations of models based on MRI are the isotropic representation of the tissue-medium and the variations in the measured electrical conductivity of brain tissue found in the literature. The conductivity of grey and white matter tissue is dependent on frequency and measurements are substantially different in the literature. Tissue conductivity from standard references such as Geddes and Baker [32] or Schwan and Kay [33] are measured in tissue samples at frequencies way beyond standard DBS frequencies. Other factors that may influence measurements performed in vivo is the tissue temperature, bleeding, leakage of CSF, decreased blood-flow due to anaesthetic agents, or increased pressure caused by the probe [34]. In addition, Hemm et al. [35] showed significant individual variations between patients during impedance measurements in the pallidum. In 1996, Gabriel at al. extracted dielectric properties of tissues from the literature of the past five decades [36]. These values together with new measurements [37] were used to described dielectric tissue properties for any frequency in the range of $10 \mathrm{~Hz}$ to $100 \mathrm{GHz}$ [38]. The data was made available online by Andreuccetti et al. [17]. In the 


\section{Influence of tissue on DBS}

heterogeneous isotropic tissue models of the present study conductivity values at $130 \mathrm{~Hz}$ from that database was used. Other groups have used different conductivity values. For bulk tissue, Yousif used conductivity values from Geddes and Baker, $\left(0.2 \mathrm{Sm}^{-1}\right)$, while the Cleveland group commonly used conductivity values from Sanses and Larson $\left(0.3 \mathrm{Sm}^{-1}\right)$ [39].

A major limitation of models based on DTI is the low image resolution in relation to the targeted anatomic regions. In addition, the performance of DTI is still limited in regions of crossing fibres because of multiple intra-voxel orientations [40]. As previously mentioned, a strong linear relationship between tissue electric conductivity and DTI was indicated by Tuch et al. [11]. However, this relationship has not been thoroughly evaluated, and in particular it has not been evaluated for different frequencies.

In addition to the tissue electrical conductivity there are other factors that may influence the outcome of DBS simulations. Butson et al. [25] evaluated the effects of various electrical tissue properties and electrode-tissue interface during DBS. Wei and Grill [41], used finite element models to study the effects of varying electrode characteristics on the current density and field distributions in an idealized electrolytic medium. Yousif et al. [26] simulated the electric field during DBS for investigation of the voltage drop at the electrode-tissue interface at the acute, transitional, and chronic post-implantation stages of DBS. Johnson and McIntyre [42] used general finite element models together with a more detailed neuron model to examined how various stimulation paradigms affected the neural output of the pallidum, and the resulting stimulation-induced network-effects 


\section{Influence of tissue on DBS}

within the basal ganglia. The influence of electrode and tissue capacitance on the volume of tissue activated have previously been studied where inclusion of a capacitance of $3.3 \mu \mathrm{F}$ had a minor effect on the stimulation waveform [24, 43]. During model-based investigations of DBS all of these factors may influence the results.

DTI is performed in some institutions in order to analyse white matter fascicles when planning trajectories during primary anatomical targeting during DBS [44]. However, a complicating factor of using DTI for patient-specific investigations is that it is not acquired on routine during DBS surgery in many clinics and therefore not available for multi-patient studies. In the present study only one patient was included. Simulations were carried out bilaterally in three different anatomic regions for three different tissue models with a total of 162 measurements. Due to the small size of the study care should be exercised in drawing general conclusion from these results. The spatial distribution of the electric field was mainly affected by the tissue within a few millimetres away from the electrode. Thus, a slight adjustment of the electrode locations would affect the results. In addition, stimulation with other electrode configurations such as bipolar or tripolar would generate a spatial distribution of the electric field different from that of monopolar stimulation. In addition, if more patients were studied the results would be affected by inter-subject anatomic variability.

In view of the anisotropic information provided by the DTI it may be suggested that DBS models based on DTI are more realistic than models based on anatomical MRI. However, this may not always be evident when the larger voxel 
volume of the DTI is taken into consideration. In the present study the voxel volume of DTI was approximately 17 times larger than for MRI (9.72 versus 0.56 $\mathrm{mm}^{3}$ ). For instance, previous studies by our group have shown that small cerebrospinal fluid (CSF) filled cystic cavities that may be visible in the MRI but not in the DTI can have substantial impact on the electric field because of the approximately 20 times larger conductivity of CSF than grey matter [45]. Thus, under such conditions tissue models based on DTI may not always be more realistic than tissue models based on MRI. Due to the known and unknown limitations patient-specific computer models based on either structural MRI or DTI may be appropriate as long as the result is considered on a rough level.

\section{Conclusions}

Heterogeneous isotropic tissue altered the spatial distribution of the electric field by up to $4 \%$ at the $0.20 \mathrm{~V} \mathrm{~mm}^{-1}$ isolevel, and up to $10 \%$ at the $0.05 \mathrm{~V} \mathrm{~mm}^{-1}$ isolevel in certain directions. Heterogeneous anisotropic tissue had a larger impact on the distribution of the electric field with an influence of up to $18 \%$ and $15 \%$ at the 0.20 and $0.05 \mathrm{~V} \mathrm{~mm}^{-1}$ isolevel, respectively. The influence of heterogeneous and anisotropic tissue on the electric field may have a clinical relevance, especially in anatomic regions that are functionally subdivided and surrounded by multiple fibres of passage such as the subthalamic area. At this point patientspecific models based on either MRI or DTI data are suitable for presenting a rough estimation of the spatial distribution of the electric field in the anatomy. 
Influence of tissue on DBS

\section{Acknowledgements}

The authors would like to thank Simone Hemm-Ode, PhD, Göran Salerud, PhD, and Mats Anderson, $\mathrm{PhD}$, for valuable input, and Eva Enqvist, $\mathrm{PhD}$, for statistical contributions. This work was financially supported by the Swedish Foundation for Strategic Research (SSF), Swedish Research Council (VR, grant number 6212008-3013), and Swedish Governmental Agency for Innovation Systems (VINNOVA, group grant number 311-2006-7661).

\section{Financial disclosure}

None of the authors reported any conflicts of interest. 


\section{References}

1. Pahwa R, Wilkinson S B, Overman J, and Lyons K E (2005) Preoperative clinical predictors of response to bilateral subthalamic stimulation in patients with Parkinson's disease. Stereotact Funct Neurosurg, 832-3: 803.

2. Isaias I U, Alterman R L, and Tagliati M (2008) Outcome predictors of pallidal stimulation in patients with primary dystonia: the role of disease duration. Brain, 131Pt 7: 1895-902.

3. Vasques X, Cif L, Gonzalez V, Nicholson C, and Coubes P (2009) Factors predicting improvement in primary generalized dystonia treated by pallidal deep brain stimulation. Mov Disord, 246: 846-53.

4. Vasques X, Cif L, Hess O, Gavarini S, Mennessier G, and Coubes P (2009) Prognostic value of globus pallidus internus volume in primary dystonia treated by deep brain stimulation. J Neurosurg, 1102: 220-8.

5. Hemm S and Wårdell K (2010) Stereotactic implantation of deep brain stimulation electrodes: a review of technical systems, methods and emerging tools. Med Biol Eng Comput, 487: 611-24.

6. Åström M, Zrinzo L U, Tisch S, Tripoliti E, Hariz M I, and Wårdell K (2009) Method for patient-specific finite element modeling and simulation of deep brain stimulation. Med Biol Eng Comput, 471: 21-8.

7. Butson C R, Cooper S E, Henderson J M, and McIntyre C C (2007) Patient-specific analysis of the volume of tissue activated during deep brain stimulation. Neuroimage, 342: 661-70.

8. Åström M, Tripoliti E, Hariz M I, Zrinzo L U, Martinez-Torres I, Limousin P, and Wårdell K (2010) Patient-Specific Model-Based Investigation of Speech Intelligibility and Movement during Deep Brain Stimulation. Stereotact Funct Neurosurg, 884: 224-233.

9. Nicholson P W (1965) Specific impedance of cerebral white matter. Exp Neurol, 134: 386-401.

10. Sotiropoulos S N and Steinmetz P N (2007) Assessing the direct effects of deep brain stimulation using embedded axon models. J Neural Eng, 42: 107-19.

11. Tuch D S, Wedeen V J, Dale A M, George J S, and Belliveau J W (2001) Conductivity tensor mapping of the human brain using diffusion tensor MRI. Proc Natl Acad Sci U S A, 9820: 11697-701.

12. Tuch D S, Wedeen V J, Dale A M, George J S, and Belliveau J W (1999) Conductivity mapping of biological tissue using diffusion MRI. Ann N Y Acad Sci, 888: 314-6.

13. Haueisen J, Tuch D S, Ramon C, Schimpf P H, Wedeen V J, George J S, and Belliveau J W (2002) The influence of brain tissue anisotropy on human EEG and MEG. Neuroimage, 151: 159-66.

14. Walckiers G, Fuchs B, Thiran J P, Mosig J R, and Pollo C (2010) Influence of the implanted pulse generator as reference electrode in finite 
element model of monopolar deep brain stimulation. J Neurosci Methods, 1861: 90-6.

15. Wakana S, Jiang H, Nagae-Poetscher L M, van Zijl P C, and Mori S (2004) Fiber tract-based atlas of human white matter anatomy. Radiology, 2301: 77-87.

16. Chaturvedi A, Butson C R, Lempka S F, Cooper S E, and McIntyre C C (2010) Patient-specific models of deep brain stimulation: influence of field model complexity on neural activation predictions. Brain Stimul, 32: 657.

17. Andreuccetti D, Fossi R, and Petrucci C (2005) Dielectric properties of body tissue. Italian National Research Council, Institute for Applied Physics, Florence, Italy http://niremf.ifac.cnr.it/tissprop/

18. Jiang H, van Zijl P C, Kim J, Pearlson G D, and Mori S (2006) DtiStudio: resource program for diffusion tensor computation and fiber bundle tracking. Comput Methods Programs Biomed, 812: 106-16.

19. Cheng D K (1989) Field and Wave Electromagnetics. Vol. ISBN 0-20152820-7. 1989: Addison-Wesley Publishing Company Inc.

20. Kindlmann G (2004) Superquadric Tensor Glyphs. Proceedings IEEE TVCG/EG Symposium on Visualization pages, May: 147-154.

21. Ennis D B, Kindlman G, Rodriguez I, Helm P A, and McVeigh E R (2005) Visualization of tensor fields using superquadric glyphs. Magn Reson Med, 531: 169-76.

22. Westin C F, Maier S E, Mamata H, Nabavi A, Jolesz F A, and Kikinis R (2002) Processing and visualization for diffusion tensor MRI. Med Image Anal, 62: 93-108.

23. Rattay F (1986) Analysis of models for external stimulation of axons. IEEE Trans Biomed Eng, 3310: 974-7.

24. Butson C R and McIntyre C C (2005) Tissue and electrode capacitance reduce neural activation volumes during deep brain stimulation. Clin Neurophysiol, 11610: 2490-500.

25. Butson C R, Maks C B, and McIntyre C C (2006) Sources and effects of electrode impedance during deep brain stimulation. Clin Neurophysiol, 1172: 447-54.

26. Yousif N, Bayford R, Wang S, and Liu X (2008) Quantifying the effects of the electrode-brain interface on the crossing electric currents in deep brain recording and stimulation. Neuroscience, 1523: 683-91.

27. Kuncel A M and Grill W M (2004) Selection of stimulus parameters for deep brain stimulation. Clin Neurophysiol, 11511: 2431-41.

28. Kuncel A M, Cooper S E, and Grill W M (2008) A method to estimate the spatial extent of activation in thalamic deep brain stimulation. Clin Neurophysiol, 1199: 2148-58.

29. Hemm S, Mennessier G, Vayssiere N, Cif L, El Fertit H, and Coubes P (2005) Deep brain stimulation in movement disorders: stereotactic coregistration of two-dimensional electrical field modeling and magnetic resonance imaging. J Neurosurg, 1036: 949-55.

30. Mikos A, Bowers D, Noecker A M, McIntyre C C, Won M, Chaturvedi A, Foote K D, and Okun M S (2011) Patient-specific analysis of the relationship between the volume of tissue activated during DBS and verbal fluency. Neuroimage, 54 Suppl 1: S238-46. 
31. Frankemolle A M, Wu J, Noecker A M, Voelcker-Rehage C, Ho J C, Vitek J L, McIntyre C C, and Alberts J L (2010) Reversing cognitivemotor impairments in Parkinson's disease patients using a computational modelling approach to deep brain stimulation programming. Brain, $133 \mathrm{Pt}$ 3: 746-61.

32. Geddes L A and Baker L E (1967) The specific resistance of biological material--a compendium of data for the biomedical engineer and physiologist. Med Biol Eng, 53: 271-93.

33. Schwan H P and Kay C F (1957) The conductivity of living tissues. Ann N Y Acad Sci, 656: 1007-13.

34. Latikka J, Kuurne T, and Eskola H (2001) Conductivity of living intracranial tissues. Phys Med Biol, 466: 1611-6.

35. Hemm S, Vayssiere N, Mennessier G, Cif L, Zanca M, Ravel P, Frerebeau P, and Coubes P (2004) Evolution of Brain Impedance in Dystonic Patients Treated by GPi Electrical Stimulation. Neuromodulation, 72: 6775(9).

36. Gabriel C, Gabriel S, and Corthout E (1996) The dielectric properties of biological tissues: I. Literature survey. Phys Med Biol, 4111: 2231-49.

37. Gabriel S, Lau R W, and Gabriel C (1996) The dielectric properties of biological tissues: II. Measurements in the frequency range $10 \mathrm{~Hz}$ to 20 GHz. Phys Med Biol, 4111: 2251-69.

38. Gabriel S, Lau R W, and Gabriel C (1996) The dielectric properties of biological tissues: III. Parametric models for the dielectric spectrum of tissues. Phys Med Biol, 4111: 2271-93.

39. McIntyre C C, Mori S, Sherman D L, Thakor N V, and Vitek J L (2004) Electric field and stimulating influence generated by deep brain stimulation of the subthalamic nucleus. Clin Neurophysiol, 1153: 589-95.

40. Landman B A, Wan H, Bogovic J A, Bazin P L, and Prince J L (2010) Resolution of Crossing Fibers with Constrained Compressed Sensing using Traditional Diffusion Tensor MRI. Proc Soc Photo Opt Instrum Eng, 7623: $76231 \mathrm{H}$.

41. Wei X F and Grill W M (2005) Current density distributions, field distributions and impedance analysis of segmented deep brain stimulation electrodes. J Neural Eng, 24: 139-47.

42. Johnson M D and McIntyre C C (2008) Quantifying the neural elements activated and inhibited by globus pallidus deep brain stimulation. $\mathrm{J}$ Neurophysiol, 1005: 2549-63.

43. Grant P F and Lowery M M (2009) Electric field distribution in a finitevolume head model of deep brain stimulation. Med Eng Phys, 319: 1095103.

44. Lemaire J J, Coste J, Ouchchane L, Caire F, Nuti C, Derost P, Cristini V, Gabrillargues J, Hemm S, Durif F, and Chazal J (2007) Brain mapping in stereotactic surgery: a brief overview from the probabilistic targeting to the patient-based anatomic mapping. Neuroimage, 37 Suppl 1: S109-15.

45. Åström M, Johansson J D, Hariz M I, Eriksson O, and Wårdell K (2006) The effect of cystic cavities on deep brain stimulation in the basal ganglia: a simulation-based study. J Neural Eng, 32: 132-8. 
Influence of tissue on DBS 